\title{
Contrast-enhanced Ultrasound Findings of Hemolymphangioma of the Kidney: The First Report in the Literature
}

\section{Hong Wang}

Sichuan University West China Hospital

Dan Yang

Sichuan University West China Hospital

Yan Luo

Sichuan University West China Hospital

Yulan Peng

west china

Wenwu Ling ( $\sim$ lingwenwubing@163.com )

Sichuan University West China Hospital

\section{Case report}

Keywords: contrast-enhanced ultrasound (CEUS), hemolymphangioma, kidney

Posted Date: August 10th, 2020

DOI: https://doi.org/10.21203/rs.3.rs-54604/v1

License: (c) (i) This work is licensed under a Creative Commons Attribution 4.0 International License. Read Full License 


\section{Abstract}

Background: Hemolymphangioma is a rare vascular and lymphatic malformation. To the author's knowledge, no cases of hemolymphangioma in the kidney have been reported in the literature.

Case presentation: We herein report a case of a 68-year-old man with low back pain. NO abnormalities were revealed in laboratory data including liver, renal function and blood and urine sediments. Ultrasonography showed a huge separated cystic mass in the upper part of left kidney. In contrastenhanced ultrasound(CEUS), the lesion of the kidney was hyper-enhanced in the hyper-enhanced in the cortical phase, and hypo-enhancement in medulla and delayed phase. Nephrectomy was performed. The pathological diagnosis was a hemolymphangioma of thekidney. His postoperative course was uneventful and he was discharged on the 12th $d$ after surgery.

Conclusions: Although rare, hemolymphangioma should be considered a differential diagnosis for masses occurring in the kidney. Complete surgical resection is the treatment of choice and affords a good prognosis.

\section{Introduction}

Hemolymphangioma is very rare, and few cases occurring in the rectum, spleen, pancreas, liver have been reported[1-6]. Hemolymphangioma often arises from congenital malformation of vascular and lymphatic system. It is believed that hemolymphangioma is a benign disease without invasive ability. Hemolymphangioma of the kidney is an uncommon disease. This report describes the first case of a hemolymphangioma of the kidney in a literature review until April 2020 (Pubmed) that were misdiagnosed based on findings from ultrasound imaging. Major symptoms in this hemolymphangioma are low back pain, and distension associated with the enlarged tumor.

\section{Case Presentation}

A 68-year-old male patient was admitted to our hospital with a history of ambiguous low back pain for the past two months. He had no medical history of abdominal trauma or operation. Physical examination revealed no abnormality of liver, renal function and blood and urine sediment analysis. He then underwent an ultrasound examination of the urinary system(Fig. 1). Gray-scale ultrasound image demonstrated a separated cystic mass in the upper part of the left kidney. The mass was approximately $9.1 \times 6.4 \mathrm{~cm}$ in size with an irregular shape and slightly unclear margin. Calcification can be seen on some septum. And some septum is thicker, the thickest is about $6 \mathrm{~mm}$. Color Doppler displayed dot-linear blood flow signals in the mass septum. For further diagnosis, the patient agreed to undergo CEUS. A $1.2 \mathrm{~mL}$ contrast agents (SonoVue, Bracco SpA, Milan, Italy) suspension was injected through his cubital vein followed by a $5 \mathrm{~mL}$ saline flush. A Resona7 ultrasound system (mindray, China) with an SC6-1U (1$6 \mathrm{MHz}$ ) transducer was used only for the examination. The mechanical index setting was 0.078 for CEUS. The depth, gain, and focus are thoroughly adjusted. Taking normal renal parenchyma as a reference, the 
contrast arrival time to the renal lesion was 14 seconds after administration of the contrast agent, and the septum of the lesion was homogeneously hyper-enhanced in the cortical phase. In the medulla and delayed phase, the lesion displayed hypo-enhancement. The combination of lesion B-Mode(separated cystic lesion) and enhancement pattern (hyper-enhancement in the cortical phase, and hypoenhancement in the medulla and delayed phase after contrast agent administration) yield a diagnosis of cystic renal carcinoma.

Laparoscopic radical left nephrectomy was performed to remove the mass, and serious adhesion between the left kidney and surrounding tissues was found. Subsequently, the left kidney adhesion release was performed. The renal mass was mainly located in the upper part of the left kidney and measured $9.0 \mathrm{~cm} \times 8.0 \mathrm{~cm} \times 7.0 \mathrm{~cm}$. The tumor cyst fluid is clear.

Microscopic examination revealed a tumor that was composed of blood and lymphatic vessels with polycystic spaces, thin wall, and dyed red lymph and blood cells within the lumen(Fig. 2).

Immunohistochemistry staining results were as follows: CD31(+), CD(34+), and ERG(+), PAX8(+), D2$40( \pm)$, and no expression for CA9(-), CK7(-)in the lesions. The final pathological diagnosis of the lesion was a renal hemolymphangioma.

No evidence of malignancy was found. The postoperative course was uneventful. The patient was discharged $12 \mathrm{~d}$ after surgery. He is currently enjoying normal life without complaints or signs of recurrence.

In this paper, ethical approval was not necessary, as this article is a case report, which is based on the clinical information of the patient. Because our case is not referred to as the patient's privacy, informed consent is not necessary, and the patient gave their permission for publication of the case.

\section{Discussion}

Hemolymphangioma is a very rare and benign tumor. Hemolymphangioma has historically demonstrated a female predilection (2.25:1 female to male) and presentation in the third to fourth decades of life[6]. It may be divided into primary and secondary lymphatic vascular tumors, with a predilection for the pancreas, spleen, and lower extremity and, less commonly, in liver lesion. The primary hemolymphangioma is considered a congenital malformation of the vascular system. The formation of this tumor may be explained by obstruction of the veno-lymphatic communication between dysembryoplastic vascular tissue and the systemic circulation. Secondary hemolymphangioma is usually caused by the injury of lymphatic vessels in trauma or operation, which induces inadequate lymph fluid drainage[7, 8]. This tumor could also invade the adjacent structures[1, 4]. In general, hemolymphangiomas are large masses of varying sized cystic cavities with thin walls, having multiple thin septa and containing fluid similar to hemorrhagic fluid, and rarely of clear lymphatic nature. The tumor size varies due to the anatomical location and relationship to the neighboring tissues[7]. Clinical symptom is non-specific, and atypical abdominal pain or compression discomfort of nearby anatomic structures is the primary reported manifestation[9]. 
Conventional US faces difficulties in determining the nature of some complicated cystic renal lesions. The insufficiency is largely because it has little ability in depicting vascularity in renal diseases. Several studies showed that the utilization of CEUS substantially improves sensitivity and specificity for renal cystic lesions[10,11]. Ultrasound contrast can show the enhancement of the solid part of cystic lesions, with high sensitivity. The irregular thickening of the cystic lesions, the nodules, the solid and cystic components in the cysts are clearly outlined. However,There are few reports of ultrasound imaging of the disease. Conventional ultrasound manifested as separated cystic mass, thickened septum (maximum about $6 \mathrm{~mm}$ ), CDFI detected dot-liner color blood flow signals at the septum. CEUS showed fast-in and fast-out enhancement pattern (hyperenhancement in the cortical phase hypoenhancement in the medullary and delayed phase), which is easy to be confused with the enhancement pattern of cystic renal cell cancer. Considering these reason, this case was misdiagnosed as cystic renal cell carcinoma.

Due to a lack of knowledge of the clinical and ultrasound, an accurate diagnosis could not usually be established preoperatively. In the case, hemolymphangioma is large in size, and blood flow signals on the septa obviously. A retrospective review of the literature found that compared with hemolymphangioma, cystic renal cell carcinoma maybe has wall nodule, more and thicker septa in ultrasonography[12]. These information may be provide useful information for ultrasound differential diagnosis. Traditional ultrasound and CEUS, combined with other imaging examinations such as CT and MRI, especially Bosniak grading of $\mathrm{CT}$, can improve the diagnostic rate of renal hemolymphangioma, and plan the surgical strategy. Nephrectomy surgery is recommended after a full evaluation of the tumor. Known as a benign tumor, there is a tendency of postoperative recurrence even after curative resection. Thus, longterm postoperative follow-up is necessary to detect the disease evolution.

\section{Conclusion}

In summary, Although hemolymphangioma of the kidney is rare and difficult to diagnose. Conventional US and CEUS may provide valuable clues to the diagnosis. It should be considered in the differential diagnosis of cystic tumors of the kidney.

\section{Declarations}

\section{Ethics approval and consent to participate}

In this present study, ethical approval was not necessary, as this article is a case report, which is based on the clinical information of the patient.

\section{Availability of data and materials}

Supporting data can be found in the Clinical Research Information System, which is the electronic medical record at the Department of Ultrasound, West China Hospital of Sichuan University. These data 
are not available publicly due to patient privacy restrictions but are available from the corresponding author upon reasonable request.

\section{Consent for publication}

The patient gave his permission for publication of the case.

\section{Funding}

The Sichuan Science and Technology Program provided all funding for the design of the study and the collection, analysis, and interpretation of the data.

\section{Authors' contributions}

Wanghong and ling wenwu assembled, analyzed, and interpreted the patient data and contributed significantly to the writing of the manuscript. SS was a major contributor in writing the manuscript. Yang dan was major contributors in coordinating patient care and writing the manuscript. All authors read and approved the final manuscript.

\section{Acknowledgements}

This research was supported by Sichuan Science and Technology Program $₫$ No. 2020YFS0211区

\section{Competing interests}

The authors declare that they have no competing interests.

\section{References}

1. Gang Chen W, Cui X-Q, Ji J-F, Du. Diffuse hemolymphangioma of the rectum: A report of a rare case[J]. World J Gastroenterol 2013 March 7; 19(9):1494-7.

2. HaiJie Hu. QiuYang Jing, FuYu Li. Hepatic Hemolymphangioma Manifesting as Severe Anemia[J]. J Gastrointest Surg. 2018;22:548-9.

3. Ye Li-FengSHui-Lin, Zhou Q-Y, Ding K-F, Qiu P-L, Deng Y-C, Zhang S-Z. Shu Zheng. A giant hemolymphangioma of the pancreas in a 20-year-old girl: a report of one case and review of the literature[J]. World Journal of Surgical Oncology 2009, 7:31 doi:10.1186/1477-7819-7-31.

4. Yoshikazu Toyoki K, Hakamada S, Narumi M, Nara D, Kudoh K, Ishidol. Mutsuo Sasaki. A case of invasive hemolymphangioma of the pancreas[J]. World J Gastroenterol. 2008;14(18):2932-4. 
5. Mei Y, Peng CiJun, Chen L, Li XiongXiong, Li W-N. DeJun Shu, WanTao Xie. Hemolymphangioma of the spleen: A report of a rare case[J]. World J Gastroenterol. 2015;21(17):5442-4.

6. Li Y, Zhang X, Pang X, Yang L. Baogan Peng. Occipitocervical Hemolymphangioma in an Adult with Neck Pain and Stiffness: Case Report and Literature Review[J]. Case Rep Med. 2017. https://doi.org/10.1155/2017/7317289.

7. Zhang X, Sheng X, Liu F, Jian R, Li L. and R. Luo.Hemolymphangioma of the chest wall: a rare case report[J]. Oncology Letters. 2012;3(4):816-8.

8. Li Y, Pang X, Yang H, Gao C, Peng B. Hemolymphangioma of the waist: a case report and review of the literature[J]. Oncology Letters. 2015;9(6):2629-328.

9. Swarup I, Bjerke-Kroll BT, Cunningham ME. Paraspinous hemolymphangioma associated with adolescent scoliosis[J]. World Journal of Clinical Cases. 2015;3(6):514-51.

10. Rübenthaler J. Mueller-Peltzer K,Negrão de, Figueiredo G, Gresser E, Clevert DA. CEUS-diagnostic workup of cystic renal lesions[J]. Radiologe.2018, 58(6):545 - 52.

11. Wei S, Tian F, Xia Q, Huang P, Zhang Y, Xia Z, Wu M, Yang B. Contrastenhanced ultrasound findings of adult renal cell carcinoma associated with Xp11.2 translocation/TFE3 gene fusion: comparison with clear cell renal cell carcinoma and papillary renal cell carcinoma[J]. Cancer Imaging. doi: 10.1186/s40644-019-0268-7.

12. Minghui S, Wang C, Jiang F, Fang X, Guo B. Diagnostic value and clinical significance of ultrasound combined with CT in cystic renal cell carcinoma[J]. ONCOLOGY LETTERS 2019,18:1395 - 401.

\section{Figures}

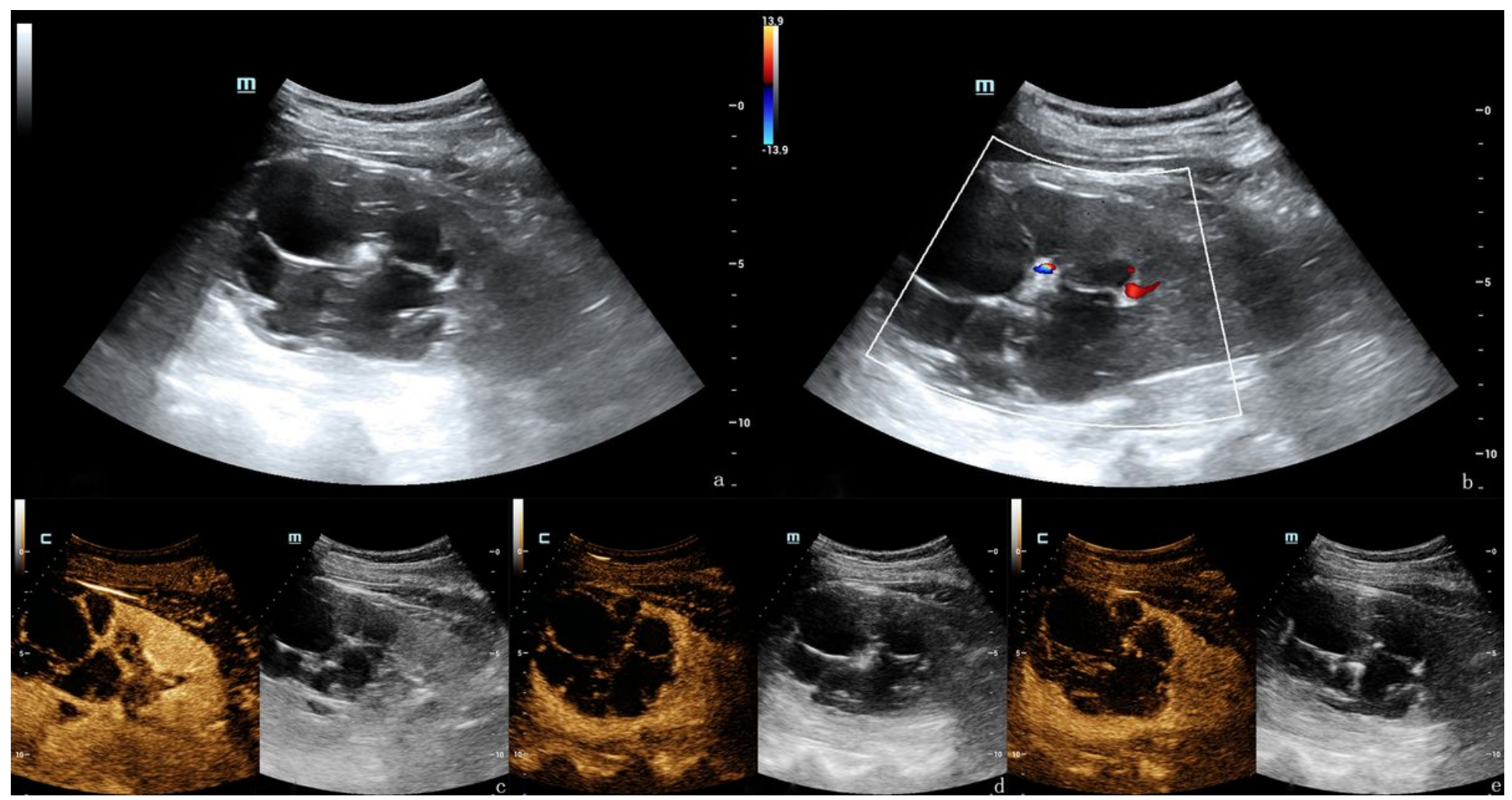




\section{Figure 1}

(a) Gray-scale ultrasound image of renal mass. The tumor is located in the upper part of the left kidney.

(b) Color Doppler displayed dot-linear blood flow signals in the mass septum. the lesion of the kidney was hyper-enhanced in the hyper-enhanced in the cortical phase (c), and hypo-enhancement in medulla (d) and delayed phase (e)

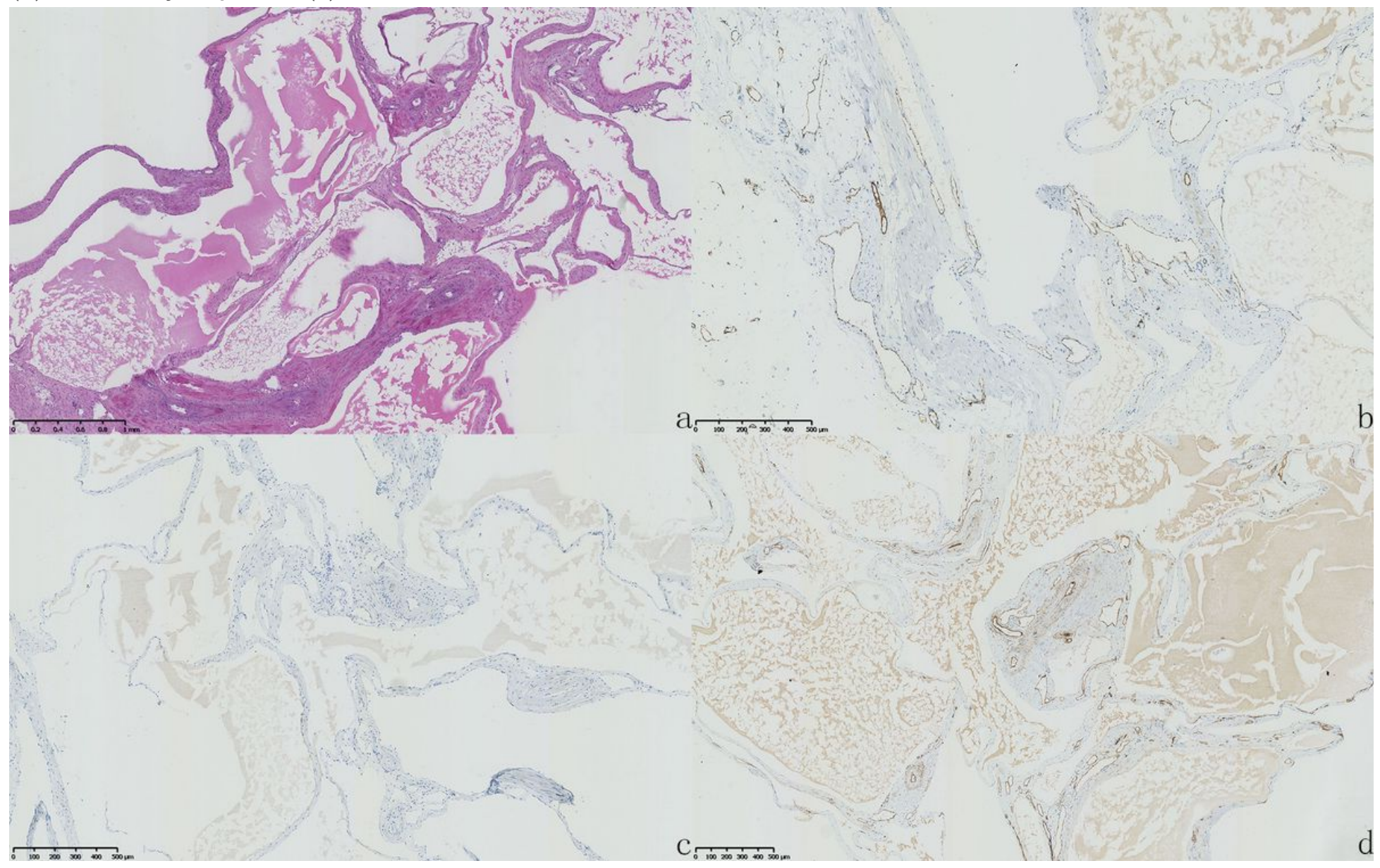

\section{Figure 2}

(a) Some dysplastic lymphatic vessels and blood vessels can be observed in the tumor. Hematoxylin and eosin stain, (HE staining, 100x). Immunohistochemical staining positive D31 (b) staining in vascular endothelial cells (100x). D2-40 (c) and CD34 (d) expressions were positive in our patient's tumor, which supported the diagnosis of hemolymphangioma of the pancreas (100x). 